\title{
Noncoherent Digital Network Coding Using Multi-tone CPFSK Modulation
}

\author{
Terry Ferrett*, Matthew C. Valenti*, and Don Torrieri ${ }^{\dagger}$ \\ ${ }^{*}$ West Virginia University, Morgantown, WV, USA. \\ ${ }^{\dagger}$ U.S. Army Research Laboratory, Adelphi, MD, USA.
}

\begin{abstract}
Digital network coding is a relaying technique that increases throughput in two-way relay networks. In contrast with analog network coding, which relays received analog signals plus noise, digital network coding relays digital codewords. The digital codewords are created by demodulation, channel decoding, and re-encoding at the relay. By using FSK and noncoherent reception, the relay may operate without knowledge of the phases of the signals transmitted by the two source terminals. In this paper, previous work on binary FSK is extended to multi-tone FSK, where the number of tones may be any power of 2. The relay receiver is formulated for any number of tones that is a power of two. Binary FSK is compared against quaternary FSK, which requires no expansion of bandwidth compared with binary FSK. The comparison is made using two metrics: the simulated bit-error rate (both with and without an outer turbo code), and the binary information rate between the sources and relay. The results illustrate that the energy-efficiency advantage of quaternary FSK on a point-to-point link is magnified when it is applied to digital network coding.
\end{abstract}

\section{INTRODUCTION}

In a two-way relay channel (TWRC), a pair of source terminals exchange information via a relay. The exchange is performed in two, three, or four time slots, depending on the protocol. When four time slots are used, the first two time slots are for the sources to communicate to the relay and the other two time slots are used for the relay to send each source its intended message. The time slots may be reduced from four to three by consolidating the last two time slots into one through the appropriate use of network coding; i.e., by having the relay broadcast the bitwise sum of the two received source packets. Using the physical-layer network coding (PNC) protocol, the first two slots are combined by having the two terminals transmit at the same time over a multiple-access channel (MAC) [1]. The relay receives the electromagnetic combination of both packets during the first slot, performs processing to compute the network coded information, and broadcasts the network codeword to the two terminals during the second slot.

PNC may be implemented in one of two ways: (1) The relay amplifies and forwards the signal received from the sources, without performing demodulation and decoding [2], which is referred to as analog network coding (ANC), or (2) The relay demodulates and decodes the received signal, and then broadcasts its estimate of the network codeword [3], which is referred to as digital network coding (DNC). Under many channel conditions, DNC offers enhanced performance over ANC. The decoding operation at the relay helps DNC remove noise from the MAC phase, while the noise is amplified by the relay when ANC is used. To contrast with DNC, we refer to the three-slot protocol as link-layer network coding (LNC) in this paper.

M.C. Valenti and T. Ferrett were sponsored by the National Science Foundation under Award No. CNS-0750821 and by the United States Army Research Laboratory under Contract W911NF-10-0109.
The simultaneous arrival of two signals at the relay complicates detection. Coherent reception is generally not feasible or very costly, since a small frequency offset between the two transmitters' oscillators will induce a rapid variation in the phase offset of the resultant signal. This problem is eliminated when using noncoherent reception, which was developed in [4]-[6] for DNC based on binary frequency-shift keying (FSK). It is well known that the capacity of FSK improves as the number of tones is increased [7]. In the present paper, we extend the prior binary system to the more general case that the number of tones may be any power of two.

Section II presents a system model that accommodates general multi-tone FSK. Section III provides the relay receiver formulated for multi-tone FSK. Section IV provides a comparison between binary FSK and quaternary FSK, which requires no expansion of bandwidth compared with binary FSK. The uncoded and coded error rates when using binary and quaternary FSK are compared for both the DNC and LNC systems. The throughput is compared by fixing the transmit signal-to-noise ratio (SNR) of the DNC and LNC system, and then determining the code rate required to achieve the required bit error rate. The selection of code rates for the latter comparison is facilitated by computing the binary information rate from the sources to the relay. Throughout the simulation study, it is assumed that the channel fading coefficients are independent from one symbol to the next; i.e., that the channel is fully-interleaved when simulating the turbo code, or ergodic when considering information rates. An example is shown for which the DNC with binary FSK yield a $37 \%$ increase in throughput over LNC. The throughput advantage increases to $41 \%$ when quaternary FSK is used.

\section{System MODEL}

The discrete-time system model is shown in Fig. 1. The source terminals $\mathcal{N}_{i}, i \in\{1,2\}$, each generate a length- $K$ information sequence, $\mathbf{u}_{i}=\left[u_{1, i}, \ldots, u_{K, i}\right]$. Each information bit $u_{k, i}$ is a member of the set $\mathcal{B}=\{0,1\}$. Each source terminal uses the same channel coding and modulation technique, denoted by the function $\Gamma_{S}(\cdot)$. A rate $r_{S}$ turbo code is used, generating a length $N_{c}=K / r_{S}$ channel codeword, denoted by $\mathbf{b}_{i}=\left[b_{1, i}, \ldots, b_{N_{c}, i}\right]$ (not shown in the diagram). Each coded bit $b_{k, i}$ is a member of $\mathcal{B}$. Let $\mathcal{D}=\{0, \ldots, M-1\}$ denote the alphabet used to select the modulated symbol, where $M$ is the modulation order. The number of bits per symbol is denoted by $\mu=\log _{2} M$. The codeword is divided into $N_{q}=N_{c} / \mu$ sets of bits. Each set of bits is mapped to an $M$-ary symbol $q_{k, i} \in \mathcal{D}$, where $k$ denotes the symbol period, and $i$ denotes the source, $i \in\{1,2\}$,

The modulated signal transmitted by source $\mathcal{N}_{i}$ during signaling interval $k T_{s} \leq t \leq(k+1) T_{s}$ is

$$
s_{k, i}(t)=\sqrt{\frac{2}{T_{s}}} \cos \left[2 \pi\left(f_{i}+\frac{q_{k, i}}{T_{s}}\right)\left(t-k T_{s}\right)\right]
$$

where $s_{k, i}(t)$ is the transmitted signal, $f_{i}$ is the carrier frequency of source $\mathcal{N}_{i}$, and $T_{s}$ is the symbol period. Eqn. (1) 


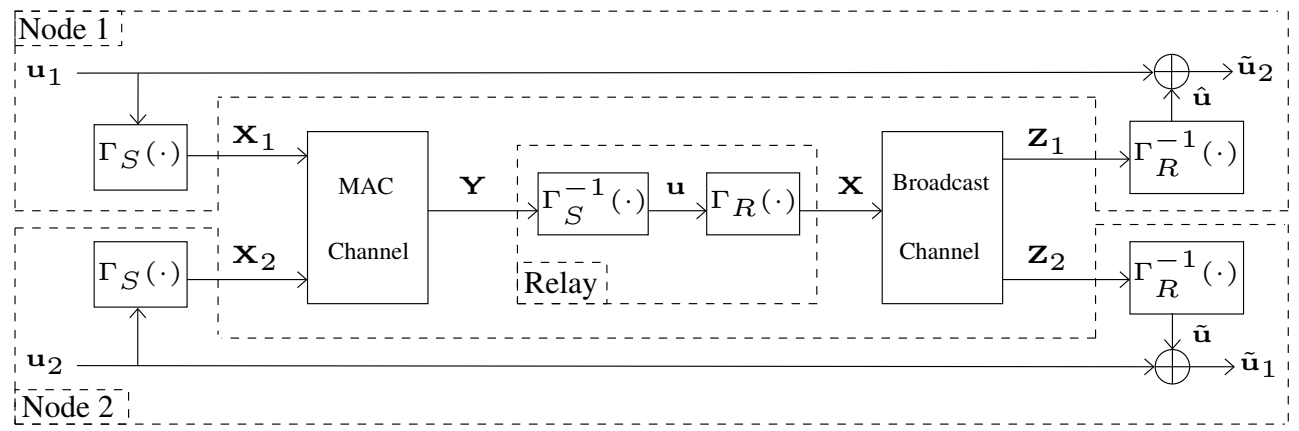

Fig. 1. Discrete-time system model.

describes continuous-phase frequency-shift keying (CPFSK) with unity modulation index, guaranteeing orthogonality under noncoherent demodulation, and having a continuous phase transition from one symbol to the next [8]. The continuoustime signals $s_{k, i}(t)$ are represented in discrete time by the set of column vectors $\left\{\mathbf{e}_{j}: j \in \mathcal{D}\right\}$. The column vector $\mathbf{e}_{j}$ is length $M$, contains a 1 at vector position $j$, and 0 elsewhere. The modulated codeword from each source is represented by the $M \times N_{q}$ matrix of symbols $\mathbf{X}_{i}=\left[\mathbf{x}_{1, i}, \ldots, \mathbf{x}_{N_{q}, i}\right]$, where $\mathbf{x}_{k, i}=\mathbf{e}_{j}$.

All channels are modeled as flat-fading channels. The channel gains are independent for every signaling interval. Let $h_{i}$ represent the complex-valued channel gain from source $\mathcal{N}_{i}$ to the relay during a single signaling interval. The gain is represented as $h_{i}=\alpha_{i} e^{j \theta_{i}}$, where $\alpha_{i}$ is the received amplitude and $\theta_{i}$ is the phase, which depends on the phase shift of the channel, the transmitter's initial phase and continuous phase constraints, and the offset between the transmitter and receiver oscillators. The energies of the transmitted signals are normalized to unity, so the power of the channel gains are selected such that the energy received by the relay from source $\mathcal{N}_{i}$ is $\mathcal{E}_{i}$, where

$$
\mathcal{E}_{i}=E\left[\left|h_{i}\right|^{2}\right]=E\left[\alpha_{i}^{2}\right] .
$$

The received signal at the relay depends on the network coding technique. Under DNC operation, information bits from both sources are received in a single signaling interval. The received signal during the $k$-th signaling interval is

$$
\mathbf{y}_{k}=h_{1} \mathbf{x}_{k, 1}+h_{2} \mathbf{x}_{k, 2}+\mathbf{n}
$$

where $\mathbf{n}$ is a zero-mean circularly-symmetric complex Gaussian random variable having covariance matrix $N_{0} \mathbf{I}_{M}$; i.e., $\mathbf{n} \sim \mathcal{N}_{c}\left(\mathbf{0}, N_{0} \mathbf{I}_{M}\right) . N_{0}$ is the one-sided noise spectral density, and $\mathbf{I}_{M}$ is the $M$-by- $M$ identity matrix. Under LNC operation, the modulated codewords from each source are received over sequential time slots, in conventional point-to-point fashion.

In the system utilizing DNC, the sources transmit simultaneously to the relay over a MAC channel. The relay receives the electromagnetic sum of interfered and faded symbols corrupted by white noise, $\mathbf{Y}=\left[\mathbf{y}_{1}, \ldots, \mathbf{y}_{N_{q}}\right]$. The relay applies the demodulation and channel-decoding function $\Gamma_{S}^{-1}(\cdot)$. The demodulation operation yields a soft estimate of the networkcoded channel codeword $\mathbf{b}=\mathbf{b}_{1} \oplus \mathbf{b}_{2}$ (not shown on the figure). Since the turbo code is a systematic linear code, $\mathbf{b}$ forms a codeword from the codebooks used by the source nodes, thus, the channel-decoding operation yields a harddecision on the network-coded message bits $\mathbf{u}=\mathbf{u}_{1} \oplus \mathbf{u}_{2}$.

In the LNC system, the sources transmit during orthogonal time slots. The received signals are demodulated independently to provide soft estimates of $\mathbf{b}_{1}$ and $\mathbf{b}_{2}$. These soft estimates are combined and turbo decoded to yield an estimate of $\mathbf{u}$ [5]. The error-rate performance during the MAC phase is better for LNC than for DNC. This is because with
LNC, the network coding operation is performed after the demodulation stage, while with DNC the network codewords are created using the combined received signal, which is added noncoherently over the MAC channel.

The relay encodes and modulates the network-coded information $\mathbf{u}$ using the function $\Gamma_{R}(\mathbf{u})$. In general, $\Gamma_{R}(\cdot)$ is different than the function $\Gamma_{S}(\cdot)$ used by the sources. The modulation technique and modulation order are the same as used at the sources, while the channel coding rate may be different. In particular, the channel code rate used by the relay is $r_{R}$, yielding a length $N_{R}=K / r_{R}$ turbo codeword. Since the end-to-end performance of the system is limited by the slot (MAC or broadcast) with highest error rate, it is important to match the error rates of the two slots. If the two sources are to transmit at the same power as the relay, then the channel code rate used by the sources in the DNC system must be lower than the rate used by the relay: $r_{S}<r_{R}$.

The relay broadcasts its encoded and modulated codeword, represented in discrete-time by the matrix $\mathbf{X}$. The signal traverses two parallel fading channels, and the source nodes, which now act as destinations, receive independently faded versions of $\mathbf{X}: \mathbf{Z}_{1}$ at $\mathcal{N}_{1}$ and $\mathbf{Z}_{2}$ at $\mathcal{N}_{2}$. The two nodes demodulate and decode their received signals using the function $\Gamma_{R}^{-1}(\cdot)$, and compute estimates of $\mathbf{u}: \hat{\mathbf{u}}$ at $\mathcal{N}_{1}$ and $\tilde{\mathbf{u}}$ at $\mathcal{N}_{2}$. Estimates of the transmitted information messages are computed as, $\tilde{\mathbf{u}}_{2}=\hat{\mathbf{u}} \oplus \mathbf{u}_{1}$ at $\mathcal{N}_{1}$ and $\tilde{\mathbf{u}}_{1}=\tilde{\mathbf{u}} \oplus \mathbf{u}_{2}$ at $\mathcal{N}_{2}$. Since the links in the broadcast phase are conventional point-to-point links with no interference, specific details of the receiver formulation will not be presented here. A detailed exposition of receiver design for turbo-coded CPFSK systems in block fading channels can be found in [9].

\section{DNC RELAY RECEIVER}

Because the relay receiver operates on a symbol-by-symbol basis, the dependence on signaling interval may be dropped to simplify the notation. Let $\mathbf{y}$ be the discrete-time received signal during the signaling interval, and let $q_{1}$ and $q_{2}$ be the symbols sent by source $\mathcal{N}_{1}$ and $\mathcal{N}_{2}$, respectively. The DNC relay receiver will map the signal received during the interval to $\log _{2}(M)$ network code bits. Let $b_{k}=b_{k}\left(q_{1}\right) \oplus b_{k}\left(q_{2}\right)$ represent the $k^{t h}$ network code bit, where $b_{k}(q)$ is the $k^{t h}$ bit in the label of symbol $q$. To facilitate soft-decision channel decoding, the relay receiver produces the log-likelihood ratio of the network-coded bit

$$
\Lambda\left(b_{k}\right)=\log \left[\frac{P\left(b_{k}=1 \mid \mathbf{y}\right)}{P\left(b_{k}=0 \mid \mathbf{y}\right)}\right], k \in\{0, \ldots, \mu-1\} .
$$

The exact form of this expression is derived in this section for several assumptions of available channel state information (CSI). The derivation proceeds in three steps: computation of the distributions of the modulated symbols received at the relay, computation of the distributions of the bits encoded by the modulated symbols, and computation of the log-likelihood ratio of the network coded bit. 


\section{A. Matched Filter Output Distributions}

In this section, the distribution of the matched-filter outputs, conditioned on the transmitted signals and available CSI, is derived for successively relaxed assumptions of channel state information.

1) Coherent Reception: When conditioned on the fading coefficients and transmitted signals, the output of the matchedfilters is the sum of two $M$-dimensional complex Gaussian vectors, which is itself Gaussian. Let $\mathbf{m}_{i, j}$ denote the mean of the received Gaussian vector when $q_{1}=i$ and $q_{2}=j$ are transmitted by the two sources. There are $M^{2}$ such vectors, which are

$$
\mathbf{m}_{i, j}=h_{1} \mathbf{e}_{i}+h_{2} \mathbf{e}_{j},(i, j) \in \mathcal{D} \times \mathcal{D}
$$

where $\mathcal{D} \times \mathcal{D}$ is the set of ordered pairs representing all possible combinations of FSK symbols transmitted by the sources. The coherent receiver must compute $p\left(\mathbf{y} \mid q_{1}=i, q_{2}=j, \mathbf{m}_{i, j}\right)$ for each $\{i, j\}$. Since the event $\left\{q_{1}=i, q_{2}=j, \mathbf{m}_{i, j}\right\}$ is equivalent to $\mathbf{m}_{i, j}$, this probability may be more compactly represented as $p\left(\mathbf{y} \mid \mathbf{m}_{i, j}\right)$. From the pdf of an M-dimensional complex-Gaussian vector, it is found that

$$
p\left(\mathbf{y} \mid \mathbf{m}_{i, j}\right)=\left(\frac{1}{\pi N_{0}}\right)^{M} \exp \left\{-\frac{1}{N_{0}}\left\|\mathbf{y}-\mathbf{m}_{i, j}\right\|^{2}\right\} .
$$

2) Noncoherent Reception with CSI: When the amplitudes of the fading coefficients are available at the receiver, but the phases are not, the conditional pdf is found by marginalizing over the unknown phase or phases of the received tone or tones. When the sources transmit different symbols $(i \neq j)$, there will be two tones received, and therefore two phases to marginalize

$$
p\left(\mathbf{y} \mid \mu_{i, j}\right)=\int_{0}^{2 \pi} \int_{0}^{2 \pi} p\left(\theta_{1}\right) p\left(\theta_{2}\right) p\left(\mathbf{y} \mid \mathbf{m}_{i, j}\right) d \theta_{1} d \theta_{2}
$$

where $\mu_{i, j}=\left|\mathbf{m}_{i, j}\right|$ is a vector whose elements are the magnitudes of the corresponding elements of $\mathbf{m}_{i, j}$ and it is assumed that the two received phases are independent. By substituting (6) into (7) and assuming uniformly distributed phases, the conditional pdf is

$$
\begin{aligned}
& p\left(\mathbf{y} \mid \mu_{i, j}\right)=\left(\frac{1}{\pi N_{0}}\right)^{M} \prod_{\substack{k=1 \\
k \neq\{i, j\}}}^{M} \exp \left\{-\frac{\left|y_{k}\right|^{2}}{N_{0}}\right\} \\
& \times\left[\frac{1}{2 \pi} \int_{0}^{2 \pi} \exp \left\{-\frac{\mid y_{i}-\alpha_{1} e^{\left.j \theta_{1}\right|^{2}}}{N_{0}}\right\} d \theta_{1}\right] \\
& \times\left[\frac{1}{2 \pi} \int_{0}^{2 \pi} \exp \left\{-\frac{\left|y_{j}-\alpha_{2} e^{j \theta_{2}}\right|^{2}}{N_{0}}\right\} d \theta_{2}\right] \\
&=\beta \exp \left\{-\frac{\alpha_{1}^{2}+\alpha_{2}^{2}}{N_{0}}\right\} I_{0}\left(\frac{2\left|y_{i}\right| \alpha_{1}}{N_{0}}\right) I_{0}\left(\frac{2\left|y_{j}\right| \alpha_{2}}{N_{0}}\right)
\end{aligned}
$$

where the factor

$$
\beta=\left(\frac{1}{\pi N_{0}}\right)^{M} \prod_{k=1}^{M} \exp \left\{-\frac{\left|y_{k}\right|^{2}}{N_{0}}\right\}
$$

is common to all possible pairs of symbols and will cancel in the log-likelihood ratio.

When the source nodes transmit the same tone $(i=j)$, only one tone will be received. The complex mean vector $\mathbf{m}_{i, j}$ will have a single nonzero entry whose value is $h=h_{1}+h_{2}=$ $\alpha e^{j \phi}$ with phase $\phi=\angle\left(\alpha_{1} e^{j \theta_{1}}+\alpha_{2} e^{j \theta_{2}}\right)$ and amplitude

$$
\alpha=\left|\alpha_{1} \exp \left(j \theta_{1}\right)+\alpha_{2} \exp \left(j \theta_{2}\right)\right| \text {. }
$$

The conditional pdf is found by marginalizing over $\phi$,

$$
p\left(\mathbf{y} \mid \mu_{i, i}\right)=\int_{0}^{2 \pi} p(\phi) p\left(\mathbf{y} \mid \mathbf{m}_{i, i}\right) d \phi .
$$

The tone is received through an equivalent fading channel with complex fading coefficient $h$. Since in Rayleigh fading $h_{1}$ and $h_{2}$ are zero-mean Gaussian, it follows that $h$ is also zero-mean Gaussian and $\phi$ is uniform. Substituting the uniform pdf of $\phi$, the conditional pdf becomes

$$
\begin{aligned}
p\left(\mathbf{y} \mid \mu_{i, i}\right) & =\left(\frac{1}{\pi N_{0}}\right)^{M} \prod_{\substack{k=1 \\
k \neq i}}^{M} \exp \left\{-\frac{\left|y_{k}\right|^{2}}{N_{0}}\right\} \\
& \times\left[\frac{1}{2 \pi} \int_{0}^{2 \pi} \exp \left\{-\frac{\left|y_{i}-\alpha e^{j \phi}\right|^{2}}{N_{0}}\right\} d \phi\right] \\
& =\beta \exp \left\{-\frac{\alpha^{2}}{N_{0}}\right\} I_{0}\left(\frac{2\left|y_{i}\right| \alpha}{N_{0}}\right)
\end{aligned}
$$

where $\beta$ is given by (9).

When the fading amplitude $\alpha$ is not known at the relay, and the amplitudes $\alpha_{1}$ and $\alpha_{2}$ are known, the receiver may approximate $\alpha$ by [5]

$$
\alpha=\sqrt{\alpha_{1}^{2}+\alpha_{2}^{2}}
$$

3) Noncoherent Reception without CSI: When the relay only has knowledge of the average received energy, the conditional pdf of the received signal is marginalized over the fading amplitudes. When the sources transmit different symbols, there are two fading amplitudes to marginalize over, and the conditional pdf becomes

$$
\begin{aligned}
p\left(\mathbf{y} \mid q_{1}=i, q_{2}=j\right) & = \\
& \int_{0}^{2 \pi} \int_{0}^{2 \pi} p\left(\alpha_{1}\right) p\left(\alpha_{2}\right) p\left(\mathbf{y} \mid \mu_{i, j}\right) d \alpha_{1} d \alpha_{2}
\end{aligned}
$$

where it is assumed that the $\alpha_{i}, i \in\{1,2\}$, are independent, each with pdf

$$
p\left(\alpha_{i}\right)=\frac{2 \alpha_{i}}{\mathcal{E}_{i}} \exp \left\{-\frac{\alpha_{i}^{2}}{\mathcal{E}_{i}}\right\} .
$$

Substituting (8) and (15) into (14) and then evaluating the integrals yields

$$
\begin{aligned}
p\left(\mathbf{y} \mid q_{1}=\right. & \left.i, q_{2}=j\right)= \\
\beta & {\left[\left(\frac{1}{\mathcal{E}_{1} \mathcal{E}_{2}}\right)\left(\frac{1}{\mathcal{E}_{1}}+\frac{1}{N_{o}}\right)\left(\frac{1}{\mathcal{E}_{2}}+\frac{1}{N_{0}}\right)\right]^{-1} } \\
& \times \exp \left\{\frac{\left|y_{i}\right|^{2} \mathcal{E}_{1}}{N_{o}\left(N_{0}+\mathcal{E}_{1}\right)}+\frac{\left|y_{j}\right|^{2} \mathcal{E}_{2}}{N_{0}\left(N_{0}+\mathcal{E}_{2}\right)}\right\} .
\end{aligned}
$$

When the same tone is transmitted by both nodes, the marginalization is over the composite fading amplitude $\alpha$, and

$$
p\left(\mathbf{y} \mid q_{1}=q_{2}=i\right)=\int_{0}^{2 \pi} p(\alpha) p\left(\mathbf{y} \mid \mu_{i, i}\right) d \alpha .
$$


As described previously, the tone is received over a fading channel with an equivalent complex-fading coefficient $h=$ $h_{1}+h_{2}$, which is zero-mean complex Gaussian. It follows that $\alpha$ is Rayleigh with pdf

$$
p(\alpha)=\frac{2 \alpha}{\mathcal{E}_{1}+\mathcal{E}_{2}} \exp \left\{-\frac{\alpha^{2}}{\mathcal{E}_{1}+\mathcal{E}_{2}}\right\} .
$$

Substituting (12) and (18) into (17) and evaluating the integral yields

$$
\begin{aligned}
p\left(\mathbf{y} \mid q_{1}=q_{2}=i\right)= & \beta\left(\frac{1}{\mathcal{E}_{1}+\mathcal{E}_{2}}\right)\left(\frac{1}{\mathcal{E}_{1}+\mathcal{E}_{2}}+\frac{1}{N_{0}}\right)^{-1} \\
& \times \exp \left\{\frac{\left|y_{i}\right|^{2}\left(\mathcal{E}_{1}+\mathcal{E}_{2}\right)}{N_{0}^{2}+N_{0}\left(\mathcal{E}_{1}+\mathcal{E}_{2}\right)}\right\} .
\end{aligned}
$$

\section{B. DNC Soft-Mapper}

The DNC Soft-Mapper (SOMAP) stage computes the distribution of the matched filter outputs conditioned on the $k^{t h}$ bit of the symbols received from the source nodes. The SOMAP takes two inputs. The first input is the set of matched filter output probabilities conditioned on all possible combinations of received symbols; i.e., $\left\{p\left(\mathbf{y} \mid q_{1}=i, q_{2}=j\right),(i, j) \in \mathcal{D} \times \mathcal{D}\right\}$, where the appropriate expression from the previous subsection is used for this conditional pdf; i.e., (8) and (12) for the receiver with CSI, and (16) or (19) for the receiver without CSI. The second input is the set of a-priori probabilities of the code bits transmitted by the source nodes, excluding the $k^{t h}$ bit; i.e., $\left\{P\left[\mathbf{b}\left(q_{1}\right) \backslash b_{k}\left(q_{1}\right)\right] P\left[\mathbf{b}\left(q_{2}\right) \backslash b_{k}\left(q_{2}\right)\right\}\right.$ where the function $\mathbf{b}(q)$ selects all code bits associated with symbol $q$.

The output of the SOMAP is the set of probabilities of matched filter outputs conditioned on the bits transmitted by the source nodes

$$
\left\{p\left(\mathbf{y} \mid b_{k}\left(q_{1}\right), b_{k}\left(q_{2}\right)\right): b_{k}\left(q_{1}\right), b_{k}\left(q_{2}\right) \in \mathcal{B} \times \mathcal{B}\right\}
$$

where $\mathcal{B} \times \mathcal{B}$ is the set of ordered pairs representing all possible combinations of bits. Each element of the output set is is related to the input sets by

$$
\begin{aligned}
& p\left(\mathbf{y} \mid b_{k}\left(q_{1}\right)=m, b_{k}\left(q_{2}\right)=n\right)= \\
& \sum_{\substack{q_{1}: b_{k}\left(q_{1}\right)=m \\
q_{2}: b_{k}\left(q_{2}\right)=n}} p\left(\mathbf{y} \mid q_{1}, q_{2}\right) P\left[\mathbf{b}\left(q_{1}\right) \backslash b_{k}\left(q_{1}\right)\right] P\left[\mathbf{b}\left(q_{2}\right) \backslash b_{k}\left(q_{2}\right)\right]
\end{aligned}
$$

\section{Network Coding Module}

The network coding module computes the log-likelihood ratio of the $k^{t h}$ network-coded bit conditioned on the matched filter outputs. The output probabilities of the SOMAP (20) form the first input to the network coding module. The a-priori probabilities of the $k^{t h}$ bits of the received CPFSK symbols form the second input.

$$
\left\{P\left[b_{k}\left(q_{1}\right)\right] P\left[b_{k}\left(q_{2}\right)\right]: b_{k}\left(q_{1}\right), b_{k}\left(q_{2}\right) \in \mathcal{B} \times \mathcal{B}\right\}
$$

Applying Bayes' rule to the output probabilities of the SOMAP (20), the probabilities of the $k^{t h}$ bits of the received symbols conditioned on the channel observations are represented as

$$
\begin{array}{r}
P\left(b_{k}\left(q_{1}\right), b_{k}\left(q_{2}\right) \mid \mathbf{y}\right)= \\
\frac{p\left(\mathbf{y} \mid b_{k}\left(q_{1}\right), b_{k}\left(q_{2}\right)\right) P\left(b_{k}\left(q_{1}\right)\right) P\left(b_{k}\left(q_{2}\right)\right)}{p(\mathbf{y})}
\end{array}
$$

Denote all possible combinations of bits transmitted by the source nodes as

1) $\mathbb{E}_{1}=\left\{b_{k}\left(q_{1}\right)=0, b_{k}\left(q_{2}\right)=0\right\}$

2) $\mathbb{E}_{2}=\left\{b_{k}\left(q_{1}\right)=1, b_{k}\left(q_{2}\right)=1\right\}$

3) $\mathbb{E}_{3}=\left\{b_{k}\left(q_{1}\right)=0, b_{k}\left(q_{2}\right)=1\right\}$

4) $\mathbb{E}_{4}=\left\{b_{k}\left(q_{1}\right)=1, b_{k}\left(q_{2}\right)=0\right\}$.

Substituting these events into Eq. (23) and combining the events as defined by Eq. (4) yields the log-likelihood ratio of the network-coded bit

$$
\Lambda\left(b_{k}\right)=\log \left[\frac{P\left(\mathbf{y} \mid \mathbb{E}_{3}\right) P\left(\mathbb{E}_{3}\right)+P\left(\mathbf{y} \mid \mathbb{E}_{4}\right) P\left(\mathbb{E}_{4}\right)}{P\left(\mathbf{y} \mid \mathbb{E}_{1}\right) P\left(\mathbb{E}_{1}\right)+P\left(\mathbf{y} \mid \mathbb{E}_{2}\right) P\left(\mathbb{E}_{2}\right)}\right] .
$$

\section{Simulation Study}

This section describes the results of simulation that demonstrates the error-rate performance at the relay and the endto-end throughput of the two-way relay channel. The performance of the LNC and DNC relay receivers is compared, considering binary and quaternary modulation. The DNC relay receiver was implemented using two types of channel state information. In one formulation, the receiver uses noncoherent reception with CSI, given by (8) and (12). The fading amplitude $\alpha$ is computed using the approximation given by (13). This CSI case is referred to as "partial CSI" in the remainder of this section. In the other formulation, the receiver operates in the absence of CSI by using (16) and (19). This case is referred to as "No CSI". Throughout this section, it is assumed that the two signals are received with equal average energy per symbol $\mathcal{E}_{s}=\mathcal{E}_{1}=\mathcal{E}_{2}$.

\section{A. Error-rate performance without an error-correcting code}

Fig. 2 shows the uncoded error-rate at the relay. Since there is no channel code, the average energy-per-bit is $\mathcal{E}_{b}=$ $\mathcal{E}_{s} / \log _{2}(M)$. While LNC outperforms DNC, the performance gap narrows with increasing modulation order. Consider the SNR required to reach an error rate of $10^{-3}$. Comparing binarry DNC and LNC with partial CSI, the required $\mathcal{E}_{b} / N_{0}$ is approximately 37 and $30 \mathrm{~dB}$, respectively, a performance gap of $7 \mathrm{~dB}$. Comparing quaternary DNC and LNC, the required $\mathcal{E}_{b} / N_{0}$ is approximately 34 and $28 \mathrm{~dB}$, respectively, a performance gap of $6 \mathrm{~dB}$. Increasing modulation order from 2 to 4 decreases the performance gap between DNC and LNC by $1 \mathrm{~dB}$.

\section{B. Error-rate performance with outer turbo code}

This section presents error-rate performance at the relay when the end nodes apply a turbo error-correcting code to their information sequences. Each end node $\mathcal{N}_{i}$ encodes its length $K=4500$ data frame into a length $N_{c}=K / r_{S}=$ $4500 / 0.9=5000$ channel codeword. The relay detects the network-coded channel codeword $\mathbf{b}=\mathbf{b}_{1} \oplus \mathbf{b}_{2}$. The relay performs turbo decoding to recover the network-coded information sequence $\mathbf{u}$.

Error-rate performance for binary and quaternary modulations orders, partial CSI, and no CSI, is shown in Fig. 3. Since a rate $r_{S}$ channel code is used, $\mathcal{E}_{b}=\mathcal{E}_{s} /\left(r_{S} \log _{2}(M)\right)$. Consider the $\mathcal{E}_{b} / N_{0}$ required to reach the error rate $10^{-3}$. In the binary case, the performance gap between DNC and LNC is approximately 6 and $8 \mathrm{~dB}$ for the partial CSI and no CSI cases, respectively. In the quaternary case, the performance gap is approximately 4 and $6 \mathrm{~dB}$, respectively. In all cases, quaternary modulation decreases the performance gap between DNC and LNC by $2 \mathrm{~dB}$. 


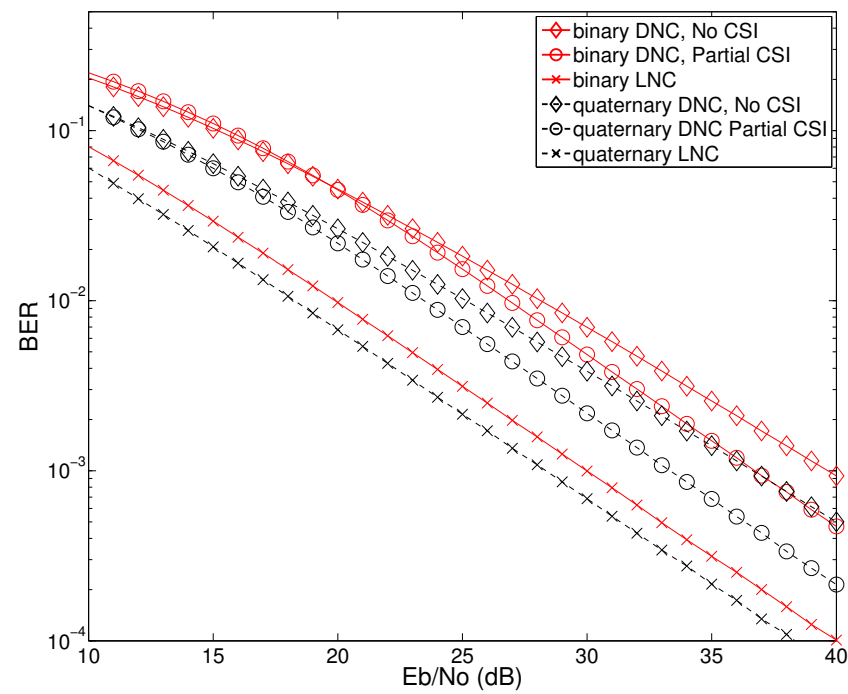

Fig. 2. Uncoded error-rate performance at the relay in Rayleigh fading.

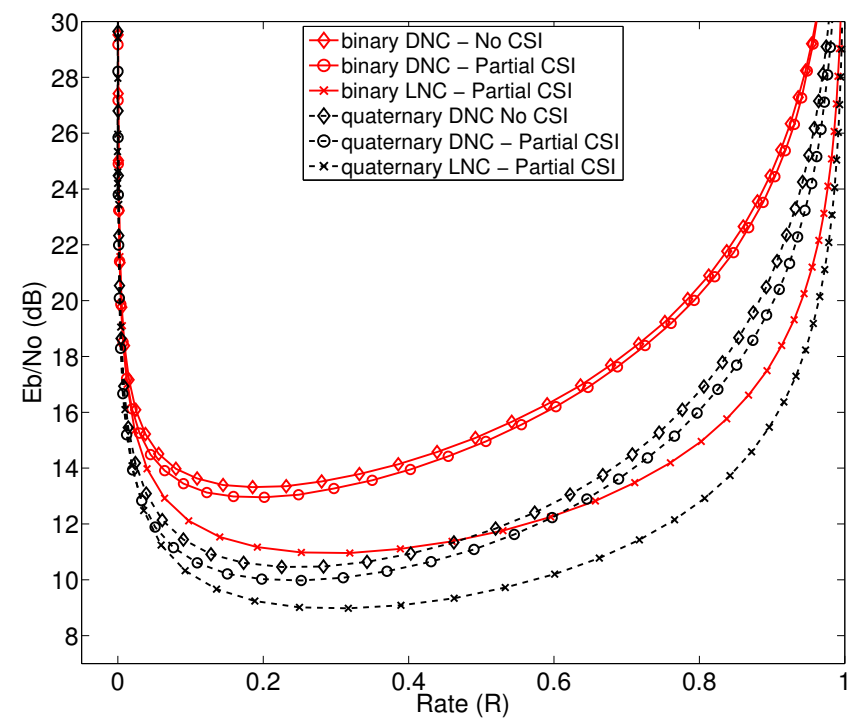

Fig. 4. Minimum $\mathcal{E}_{b} / N_{0}$ (in $\mathrm{dB}$ ) required for $\mathrm{LNC}$ and DNC to achieve a binary information rate $R$, for binary and 4-ary modulation, no CSI, and partial CSI.

\section{Binary information rate}

The throughput of DNC and LNC can be compared by equalizing the error rate of both systems through the selection of appropriate channel code rates, and comparing the rates. An estimate of the code rates that yield approximately equal error-rate performance at the relay for both DNC and LNC can be found by computing the binary information rate, which is the mutual information between the bits at the input of the modulator and the bit LLR's at the output of the demodulator. A Monte Carlo simulation procedure for computing binary information rate is given in [7], which refers to the binary information rate as the bit-interleaved coded modulation (BICM) capacity. The procedure computes simulated values of

$$
R=1-\frac{\log _{2}(e)}{\mu} \sum_{k=0}^{\mu-1} E\left[\max *\left(0, \Lambda\left(b_{k}\right)(-1)^{b_{k}\left(q_{1}, q_{2}\right)}\right)\right] .
$$

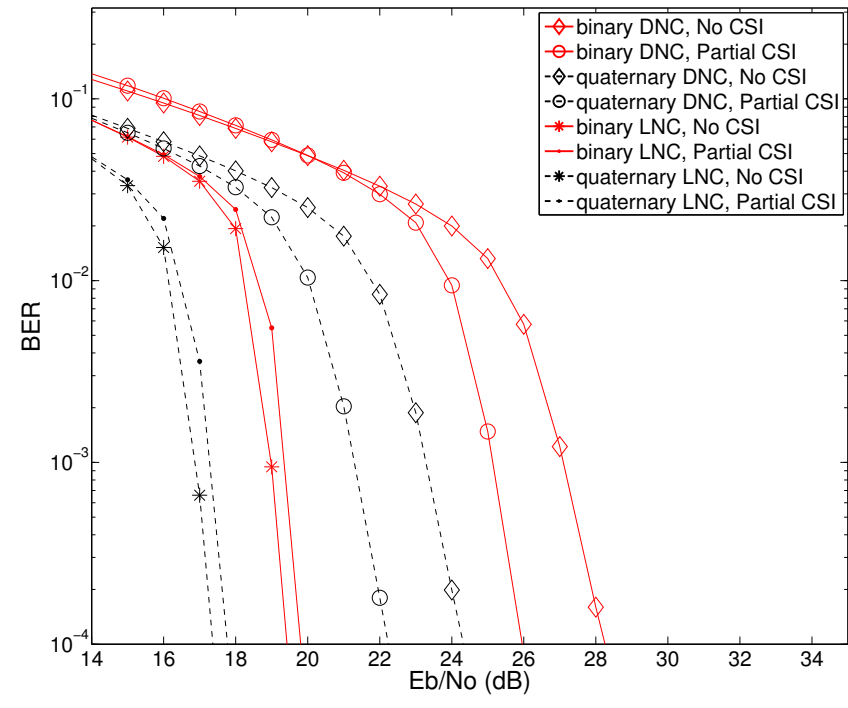

Fig. 3. Coded error-rate performance at the relay in Rayleigh fading, using Turbo code rate $r_{S}=4500 / 5000$. Modulation orders 2 and 4 are considered.

where $\Lambda\left(b_{k}\right)$ is the log-likelihood ratio defined by Eq. (24), for the cases of both partial CSI and no CSI. Further, $b_{k}\left(q_{1}, q_{2}\right)$ denotes the $k^{t h}$ network coded bit formed from the symbols $q_{1}$ and $q_{2}$ transmitted by the source nodes during a single signaling interval. Finally, the operator $\max *(\cdot)$ is defined as $\max *(x, y)=\max (x, y)+\log \left(1+e^{-|x-y|}\right)$, where $\log (\cdot)$ is taken to the base $e$.

For both the DNC and LNC systems, the binary-information rate was estimated using a Monte Carlo procedure. The binary input bits $b_{k}$ were selected at random and with equal probability and used to select the transmitted signals with an energy per symbol of $\mathcal{E}_{s}$ over a simulated AWGN channel. For each receiver formulation described in Section III, the bitwise LLRs $\Lambda\left(b_{k}\right)$ were computed for each input bit. Finally, the rate was found using (25), where the expectation is taken over a large number of Monte Carlo trials (one million per value of $\left.\mathcal{E}_{s} / N_{0}\right)$. After computing $R$, the minimum value of $\mathcal{E}_{b} / N_{0}$ can be found by plotting $\mathcal{E}_{b} / N_{0}=\mathcal{E}_{s} / N_{0} / R$ as a function of $R$.

Fig. 4 shows the minimum $\mathcal{E}_{b} / N_{0}$ required to achieve rate binary information rate $R$. The curves demonstrate the superior energy efficiency of quaternary FSK modulation. As displayed, it appears that LNC is superior to DNC. However, recall that LNC requires two time slots during the MAC phase, while DNC does not. To compare the two systems on the basis of throughput, rates that require the same value of $\mathcal{E}_{b} / N_{0}$ should be selected. This can be found by selecting a desired operating value of $\mathcal{E}_{b} / N_{0}$, which forms a horizontal cut across the figure. Select a curve for DNC and a curve for LNC that operates under the same CSI assumptions. By finding where the two curves intersect the cut, the two operating rates can be determined.

As an example, consider a target $\mathcal{E}_{b} / N_{0}=16 \mathrm{~dB}$ for binary DNC and LNC, assuming partial CSI. According to Fig. 4, the LNC system requires a code rate of approximately 0.9 , and the DNC system requires a code rate of approximately 0.7. This procedure is used to select the channel code rates which equalize error-rate performance in the following throughput comparison.

\section{Throughput comparison - DNC and LNC}

To compare the throughputs of DNC and LNC, the code rates of the two systems should be selected to provide roughly the same end-to-end error rates. The selection is guided by 
TABLE I

CODE RATES ACHIEVING DNC CAPACITY AT THE RELAY @ 16 DB

\begin{tabular}{|c|c|c|}
\hline CSI & $\mathbf{M = 2}$ & $\mathbf{M}=\mathbf{4}$ \\
\hline None & $4500 / 6500 \approx 0.70$ & $4500 / 5940 \approx 0.75$ \\
\hline Partial & $4500 / 6300 \approx 0.71$ & $4500 / 5640 \approx 0.79$ \\
\hline
\end{tabular}

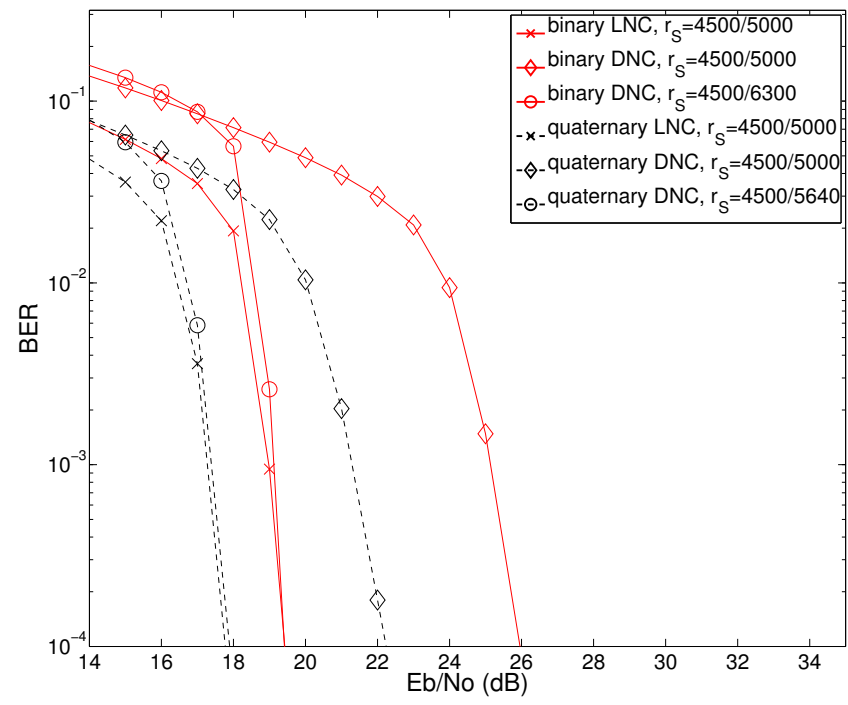

Fig. 5. Coded error-rate performance used to compare DNC and LNC throughput, assuming partial CSI is available.

using the binary information rates given in Fig. 4. For instance, suppose that LNC is used with a rate $R=0.9$ code, and the corresponding DNC code rates need to be determined. From Fig. 4, it can be seen that the $\mathcal{E}_{b} / N_{0}$ required by binary LNC (with partial CSI) to achieve $R=0.9$ is $\mathcal{E}_{b} / N_{0}=17.8$ $\mathrm{dB}$. At the same value of $\mathcal{E}_{b} / N_{0}$, the DNC system achieves $R=0.70$ without CSI and $R=0.71$ with partial CSI. Similarly, quaternary LNC requires $\mathcal{E}_{b} / N_{0}=15.6 \mathrm{~dB}$ to achieve $R=0.9$. At the same value of $\mathcal{E}_{b} / N_{0}$, the DNC system achieves $R=0.75$ without CSI and $R=0.79$ with partial CSI. Using this procedure, rates for all the DNC systems considered were found and are listed in Table I. Since DNC and LNC implement the broadcast phase in the same way, a common channel code rate of $r_{R}=4500 / 5000$ is used for all systems in the broadcast phase.

Error-rate curves showing the performance of LNC and DNC using the rates listed in Table I are shown in Fig. 5 for the partial CSI case. Also shown in the figure is a curve for the DNC system using rate $r_{s}=4500 / 5000$; i.e., the same rate used by the LNC system. The curves show that the binary LNC system with rate $r_{s}=4500 / 5000$ provides the same performance as the binary DNC system with rate $r_{s}=4500 / 6300$. Similarly, it shows that the quaternary LNC system with rate $r_{s}=4500 / 5000$ provides the same performance as the quaternary DNC system with rate $r_{s}=4500 / 5640$. The agreement of these curves confirms the validity of using the binary information rate to select appropriate code rates. A similar plot was produced for the no-CSI case, but is not shown due to space limitations. With the selected code rates, DNC and LNC have the same error rate performance in the broadcast phase, yielding a similar end-to-end error rate.

Having selected code rates that provide similar end-to-end performance, a comparison of the throughputs of the DNC and LNC systems can be made. The distinction between the two systems is, of course, in the MAC phase. Let $N_{L}$ and $N_{D}$ indicate the number of channel-coded bits sent by each source
TABLE II

THROUGHPUT IMPROVEMENT - DNC OVER LNC

\begin{tabular}{|c|c|c|}
\hline \multicolumn{3}{|c|}{ Throughput Improvement $-T_{P}$} \\
\hline CSI & $\mathbf{M = 2}$ & $\mathbf{M}=\mathbf{4}$ \\
\hline None & $30.4 \%$ & $32.7 \%$ \\
\hline Partial & $37.1 \%$ & $41.0 \%$ \\
\hline
\end{tabular}

terminal for the LNC and DNC systems, respectively. Because LNC requires that the two sources transmit in orthogonal time slots, the duration of the MAC phase is $2 N_{L} / \log _{2}(M)$ symbol periods. With the DNC system, the two sources transmit at the same time and the duration of the MAC phase is $N_{D} / \log _{2}(M)$ symbol periods. The broadcast phase for both systems requires the same amount of time. Letting $N_{B}$ indicate the number of channel-coded bits sent during the broadcast phase, the duration is $N_{B} / \log _{2}(M)$. The total number of bits transferred by each system is $2 K$, and the throughput of each (in bits per second) is found by dividing $2 K$ by the duration of the entire transfer; i.e., the sum of the MAC and broadcast phases. The ratio of the throughput of the DNC system to that of the LNC system is:

$$
T_{F}=\frac{2 K \log _{2}(M) / T_{s}\left(N_{D}+N_{B}\right)}{2 K \log _{2}(M) / T_{s}\left(2 N_{L}+N_{B}\right)}=\frac{2 N_{L}+N_{B}}{N_{D}+N_{B}}
$$

and the percentage improvement is $T_{P}=\left(T_{F}-1\right) \times 100$. For purposes of comparison, $N_{B}=N_{L}=5000$, and the values of $N_{D}$ are the denominators in Table I. The throughput improvements for modulation orders 2 and 4 , with partial CSI and no CSI, are summarized in Table II.

\section{CONClusion}

DNC increases throughput in the two-way relay network, but is costly to implement using coherent reception. The technique is well-suited for FSK systems, and it is known that the capacity of FSK increases with the number of tones. In this paper, previous work considering DNC using binary FSK is extended to $M$-ary FSK, where $M$ is a power of two. Special attention was paid to the case of $M=4$, as bandwidth efficiency is the same as the binary case. It is shown that DNC improves throughput over LNC in a typical application, and the improvement increases from $37.1 \%$ for $M=2$ to $41 \%$ for $M=4$.

\section{REFERENCES}

[1] S. Fu, K. Lu, Y. Qian, and H.-H. Chen, "Cooperative wireless networks based on physical layer network coding," IEEE Wireless Commun. Mag., pp. 86-95, Dec. 2010.

[2] S. Katti, S. Gollakota, and D. Katabi, "Embracing wireless interference: analog network coding," Proc. ACM SIGCOMM, pp. 397-408, 2007.

[3] S. Zhang, S. C. Liew, and P. P. Lam, "Hot topic: physical-layer network coding," Proc. 12th Annu. Int. Conf. Mobile Comput. and Netw., pp. 358365, 2006.

[4] J. Sørensen, R. Krigslund, P. Popovski, T. Akino, and T. Larsen, "Physical layer network coding for FSK systems," IEEE Commun. Lett., vol. 13, no. 8, pp. 597-599, Aug. 2009.

[5] M. C. Valenti, D. Torrieri, and T. Ferrett, "Noncoherent physical-layer network coding using binary CPFSK modulation," Proc. IEEE Military Commun. Conf., Oct. 2009.

[6] _ , "Noncoherent physical-layer network coding with FSK modulation: Relay receiver design issues," IEEE Trans. Commun., vol. 59, Sept. 2011.

[7] M. C. Valenti and S. Cheng, "Iterative demodulation and decoding of turbo coded $M$-ary noncoherent orthogonal modulation," IEEE J. Select. Areas Commun., vol. 23, pp. 1738-1747, Sept. 2005.

[8] J. G. Proakis and M. Salehi, Digital Communications, 5th ed. New York, NY: McGraw-Hill, Inc., 2008.

[9] S. Cheng, M. C. Valenti, and D. Torrieri, "Robust iterative noncoherent reception of coded FSK over block fading channels," IEEE Trans. Wireless Commun., vol. 6, pp. 3142-3147, Sept. 2007. 